\section{FRI0203 PULMONARY INVOLVEMENT IN JUVENILE SCLERODERMA}

AM Romicka, A Malinowski. Pediatric Clinic, Institute of Rheumatology, Warsaw, Poland

10.1136/annrheumdis-2001.284

Background Scleroderma in children manifests more frequently in the form of localised disease than syptemic one. This disorder is characterised by the presence of hard and tight skin (linear, localised and generalised morphea); systemic sclerosis, besides the skin changes it manifests the internal organ involvement.

Objectives The aim of this report was an assessment of the frequency and nature of lung lesions in children with scleroderma. Methods The study comprised 32 non selected pts, aged from 4 to 18 yrs., 16 suffering from localised and 16 from systemic scleroderma. The disease duration ranged from 6 months to $14 \mathrm{yrs}$. The relation between clinical symptoms, $\mathrm{x}-$ ray findings and functional pulmonary tests were performed.

Results Clinical and/or radiological signs (cought, dyspnoe, interstitial thickening) were found in 9 pts. The results of the functional investigation showed an impairment of the pulmonary function in $80.6 \%$ of pts with systemic sclerosis and $50 \%$ - with localised scleroderma, (total 65.6\%). A decrease in DLCO (in 1/ 3 of pts in association with restrictive ventilatory failure) belonged to the most frequent of the lung damages.

Conclusion

- Pulmonary involvement is frequent, however not a main feature in the course juvenile scleroderma.

- Abnormalities in functional tests were often the only manifestation of the respiratory sys-tem involvement.

- Lung functional disturbances may be independent of clinical features, muscular on skin involvement.

- Respiratory functional tests are helpful in estimation of systemic scleroderma in the cases manifesting only the clinical form of localised one.

\section{REFERENCES}

1 Chomette G, Tranbaloc P, Beaufils H, et al. Les sclerodermies viscerales. Ann Med Interne (Paris) 1984;135:606

2 Greenwald Gl, Tashkin DP, Gong $\mathrm{H}$, et al. Longitudinal changes in lung function and respiratory symptoms in progressive systemic sclerosis. Am J Med. 1987;83:83

3 Lahman TJA. Systemic and localized scleroderma in children. Curr Opin Rheumatol. 1995;8:576

4 Uziel Y, Miller H, Lazar RM. Scleroderma in children. Pediatr Clin North Am. 1995:42:1171

5 Deheu I, Roujcan J-C, Cosnes A. Revuz: internal involvement in localized scleroderma. Medicine (Baltimore) 1994:73:241

6 Laxer RM, Feldman BM. General and local scleroderma in children and dermatomyositis and associated syndromes. Curr Opin Rheumatol. 1997;9:458

\section{FRI0204 TRIGEMINAL SENSORY NEUROPATHY IN SYSTEMIC SCLEROSIS}

YV Grachov, NG Guseva, VM Grinin, ML Kukushkin, AV Syrovegin, VF Guravlev. Clinical Department, Institute of Rheumatology of RAMS, Moscow, Russia

\subsection{6/annrheumdis-2001.285}

\section{Background}

Objectives Systemic sclerosis (SSc) is one of the few somatic disorders which induce isolated or combined trigeminal sensory neuropathy (TSN).

Methods A screening of 101 patients with SSc hospitalised at the Institute of Rheumatology over a period of six months in 2000 revealed that eight patients $(7,9 \%)$ had TSN (all of them were females aged 42-69 with a clinical history of SSc of 1 to
12 years). In two cases, SSc was associated with the SJÖGREN'S syndrome; one case was associated with polymyositis. All the patients with TSN underwent rheumatological and neurological examinations including the recording of blink reflex.

Results The main clinical manifestation of TSN was bilateral asymmetric numbness in all the three divisions of the trigeminal nerve, especially in the perioral area. Five patients had qualitative taste disorders including «oral astereognosis», i.e., monotypical sensations in the oral cavity on taking up foods of different kinds and tastes. In two cases, TSN was manifested as constant and constant or «neuralgia-like» facial pains. Irrespective of remissions (which could be spontaneous), in each case facial pain was one of the most significant manifestations of SSc. All the patients had disturbances in surface sensitivity (hyper- or hypoesthesia) in the distal parts of the upper and lower extremities. In three cases, early symptomes of TSN (e.g., facial paraesthesia, numbness and hyperesthesia) developed simultaneously with the changes in the skin colour (especially of fingers), skin oedema, arthralgias and malaise. In the rest of the patients, TSN developed within 6 months to 3 years after the Raynaud's syndrome, sclerodactyly and other manifestations of SSc. The blink reflex records revealed modest prolongation of ipsilateral R1 responses. Specific therapy of TSN included administration of vasoactive drugs (e.g., pentoxifylline, vazaprostan) and group B vitamins. The elimination of facial pain presented a problem. The use of NSAID resulted in short-term attenuation of pain; in one case, topical application of ibuprofen (in the form of a cream) was more effective. Patients with paroxysmal manifestations of pain received additionally carbamazepine $(300-400 \mathrm{mg} / \mathrm{d})$. The efficiency of physio- and reflexotherapy in patients with acute facial pain syndrome is open to question.

Conclusion TSN is a typical neurological sign of SSc (with frequency $7,9 \%$ of cases), which is manifested as facial numbness, qualitative taste disorders including "oral astereognosis», pain and paraesthesiae. Most often, TSN develops within 6 months to 3 years after the appearance of other characteristic features of SSc. A combination of bilateral sensory disturbances in the facial area and distal parts of the upper and lower extremities suggests that TSN can be regarded as a partial form of generalised sensory polyneuropathy.

\section{FRI0205 CLUES TO SYSTEMIC SCLEROSIS SINE SCLERODERMA}

${ }^{1}$ I Rosner, ${ }^{2} \mathrm{G}$ Slobodin, ${ }^{1} \mathrm{M}$ Rozenbaum, ${ }^{2} \mathrm{JE}$ Naschitz, ${ }^{3} \mathrm{E}$ Toubi, ${ }^{3} \mathrm{~A}$ Kessel, ${ }^{2} \mathrm{D}$ Yeshurun. ${ }^{1}$ Rheumatology; ${ }^{2}$ Internal Medicine A; ${ }^{3}$ Immunology, Bnai Zion Medical Center, Haifa, Israel

10.1136/annrheumdis-2001.286

Background There is substantial difficulty in the diagnosis of progressive systemic sclerosis where visceral involvement precedes cutaneous expression of the disease.

Objectives To describe clues to the diagnosis of progressive systemic sclerosis sine scleroderma (ssSSc).

Methods Report of 3 cases and review of the MEDLINE database concerning ssSSc.

Results Tweny five patients from the literature and 3 from our unit fulfilled the inclusion criteria, 13 females and 15 males, mean age 51.2(SD17.2) years. Conditions motivating the search for ssSSc were pulmonary fibrosis (44\%), congestive heart failure (19\%), esophageal dysfunction (7\%), malabsorption (11\%), nephropathy (11\%), fever of unknown origin $(3.5 \%)$ and seizures $(3.5 \%)$. An overt undifferentiated connective tissue disease was present in 7 pts. Multisystem involvement became apparent 
when carefully searched for in all but one patient. ANA and RF were found in 13 of 17 and "scleroderma pattern" was noted in 9 of 13 patients on nailfold capillaroscopy. Taut skin developed later in $57 \%$ of pts following extracutaneous presentation by 1 month to 7 yrs (mean 2.3 yrs).

Conclusion Clues to the diagnosis of ssSSc can be classified as: 1) background conditions - unclassified system disorder, Raynaud's, polyarthritis 2) evolving visceropathy of obscure provenience - dysphagia, malabsoption, pulmonary fibrosis, heart failure, nephropathy 3) corroborative laboratory and clinical features - hyper/hypopigmentation, telangiectasis, calcinosis, typical nailfold capillaroscopy 4) confirmatory evidence - visceral biopsy, appearance of taut skin.

\section{FRI0206 PLASMA HOMOCYSTEINE LEVELS IN PATIENTS WITH SYSTEMIC SCLEROSIS}

${ }^{1} \mathrm{~T}$ Pirildar, ${ }^{2} \mathrm{~N}$ Turgan, ${ }^{1} \mathrm{E}$ Tunc, ${ }^{1} \mathrm{~T}$ Turk, ${ }^{2} \mathrm{D}$ Ozmen, ${ }^{1} \mathrm{G}$ Keser, ${ }^{2} \mathrm{O}$ Bayindir, ${ }^{1} E$ Doganavsargil. ${ }^{1}$ Rheumatology; ${ }^{2}$ Clinical Biochemistry, Ege University, Izmir, Turkey

10.1136/annrheumdis-2001.287

\section{Background}

Objectives Homocysteine is a nonessential amino acid containing sulfur. Homocysteine has a toxic effect on the endothelium suggesting that it may take part in the etiopathogenesis of the diseases with vascular impairment. Since complex etiopathogenesis of systemic sclerosis (SSc) involves endothelial injury and activation, elevated plasma homocysteine levels might be expected in this disease. Standing from this point, we investigated plasma homocysteine levels in patients with limited (ISSc) and diffuse (dSSc) systemic sclerosis and in healthy controls.

Methods Serum samples were collected from 59 (31 limited and 28 diffuse; F/M:56/3 male) patients with systemic sclerosis attending Ege University Rheumatology department, and 27 age and sex matched healthy controls were included in the study. Homocyteine was measured by high-performance liquid chromotography. Hyperhomocyteinemia may occur due to diabetes mellitus, hyperlipidemia and vitamin B12, folic acid deficiencies. Therefore, patients with these conditions excluded from the study.

Results Statistical analysis was performed using one way analysis of varience test. All data are presented as mean $=S D$. A p value $<0.05$ was accepted as statistically significant.

Plasma homocyteine levels in patients with diffuse SSc (16.31 $\pm 5.916 \mathrm{mmol} / \mathrm{L})$ and limited SSc $(13.27 \pm 8.352 \mathrm{mmol} / \mathrm{L})$ were significantly higher than healthy controls $(6.34 \pm 1.540$ $\mathrm{mmol} / \mathrm{L}$ ). When we compared the two subgroups of SSc, plasma homocystein levels in diffuse SSc, was significantly higher than limited SSc $(\mathrm{F}=19.22 \mathrm{p}<0.001)$.

Conclusion Numerous clinical studies have been published on the association between homocysteine and vascular diseases. It has been shown to be cytotoxic to endothelium, to induce a vascular-endothelial cell activator, and to promote smooth muscle cell proliferation. Our finding of

Significantly higher plasma homocysteine levels in the both subgroups of SSc implicate the contribution of hyperhomocysteinemia to endothelial damage in this disease.

\section{FRI0207 ASSESSMENT OF TRIO AS THE CANDIDATE GENE FOR FAMILIAL CHONDROCALCINOSIS CCAL2}

${ }^{1}$ A Pendleton, ${ }^{1} \mathrm{GD}$ Wright, ${ }^{2} \mathrm{MD}$ Doherty, ${ }^{3} \mathrm{R}$ Shiang, ${ }^{3} \mathrm{AE}$ Hughes. ${ }^{1}$ Rheumatology, Musgrave Park, Belfast, UK; ${ }^{2}$ Academic Rheumatology, University of Nottingham, Nottingham, UK; ${ }^{3}$ Medical Genetics, Queens University Belfast, Belfast, UK

\subsection{6/annrheumdis-2001.288}

Background Chondrocalcinosis is usually caused by the deposition of calcium pyrophosphate crystals on articular cartilage. There is a clear relationship between this crystal deposition and excess extracellular triphosphate and pyrophosphate. TRIO, a novel multidomain transmembrane protein which promotes the exchange of guanine diphosphate for guanine triphosphate in the extracellular space is an excellent positional candidate gene for CCAL2.

Objectives

Methods A positional candidate gene strategy was employed as a valid approach to identify the putative disease locus for CCAL2. The Online Mendelian Inheritance in Man OMIM cytogenetic map was used to identify TRIO as a possible candidate gene within the critical region on chromosome $5 \mathrm{p}$. Mutation analysis required the design of PCR primers to amplify the coding DNA from human genomic DNA in affected individuals. The coding DNA sequence for TRIO was known ${ }^{1}$ but information on the intron-exon splicing sequences was unavailable. Sotgun sequence obtained from a P1-derived artificial chromosome (PAC) 514p4 from a PAC contig of the critical region had shown homology with TRIO using the homology search algorithms BLASTN. The intron-exon junctions were identified from this PAC and other adjacent PACs on the contig, using a combination of vectorette PCR and the Genome Priming System (New England Biolabs inc) followed by Big Dye Terminator sequencing on an $377 \mathrm{ABI}$ genetic analyser.

Results From the TRIO coding DNA sequence of 9 kilobases over 55 exons were identified. Both of the functional guanine exchange factor (GEF) domains (18 exons) have been fully sequenced but no mutations have yet been identified in affected members from two large families.

Conclusion TRIO is a novel multidomain transmembrane protein which is localised within the CCAL2 region on chromosome $5 \mathrm{p}$. It is likely to influence the availability of extracellular triphophate and thus pyrophosphate through the function of the active GEF domains. This gene however has no mutations within the coding regions for both the GEF domains in two large kindreds with familial chondrocalcinosis.

\section{REFERENCE}

1 Debant A, Serra-Pages C, Seipel K, O'Brien S, Tang M, Park S-H, et al. The multidomain protein Trio binds the LAR transmembrane tyrosine phosphatase, contains a protein kinase domain, and has a separate rac-specific and rho-specific guanine nucleotide exchange factor domains. Proc Natl Acad Sci USA 1996;93:5466-71

\section{FRI0208 COEXPRESSION OF TH1 AND TH2-ASSOCIATED CHEMOKINE RECEPTORS IN T CELLS INFILTRATING EARLY FIBROTIC SKIN IN A SLE PATIENT}

${ }^{1} \mathrm{C}$ Chizzolini, ${ }^{2} \mathrm{M}$ Hess, ${ }^{2} \mathrm{MG}$ Uguccioni, ${ }^{2} \mathrm{M}$ Baggiolini, ${ }^{1} \mathrm{JM}$ Dayer, ${ }^{2} \mathrm{P}$ Loetscher. ${ }^{1}$ Division of Immunology and Allergy, Geneva University Hospital, Geneva, Switzerland; ${ }^{2}$ Theodor Kocher Institute, Bern University, Bern, Switzerland

10.1136/annrheumdis-2001.289

Background Fibrotic skin changes in systemic sclerosis (SSc) and allied conditions characteristically are preceded by an 